\title{
Exploration and Analysis of Cyberpunk on the Self- identification of Juvenile from the Perspective of Visual Culture
}

\author{
Qianchen Cheng ${ }^{1, *}$ Kaili Xie ${ }^{1}$ Shuchao Chen ${ }^{1}$ \\ ${ }^{1}$ Xiamen Academy of Arts \& Design, Fuzhou University, Xiamen, Fujian 361000, China \\ *Corresponding author. Email: 496317816@qq.com
}

\begin{abstract}
In the era of visual culture and the Internet media nowadays, Cyberpunk, as a kind of youth subculture, often features strong identification and distinct visual and cultural characteristics. It has given rise to the formation of large interest groups on the Internet, including a large number of young people. This paper intends to explore the positive and negative effects of Cyberpunk on teenagers' self-identification from the perspective of visual culture on the basis of the forms and features of manifestation, as well as the spiritual core of the Cyberpunk culture and the characteristics of teenagers' self-identification, and designs a strategy to reasonably deal with the spread of the Cyberpunk in visual culture.
\end{abstract}

Keywords: Youth subculture, Visual culture, Cyberpunk, Self-identification.

\section{INTRODUCTION}

In recent years, the movies Battle Angel: Alita, Blade Runner 2049 and the animation PsychoMeasurer have set off rounds of cyberpunk fever. In the field of games, PC games such as Neil . Mechanical Age, Cyberpunk 2077, etc., and mobile games such as War Double Punishing, Transistor and other excellent games have caused a strong response on the Internet. Among them, the PC-side game Cyberpunk 2077, which has not yet been released, attracted nearly 500,000 netizens to watch it simultaneously during its live broadcast on Twitch on August 29, 2019. In addition, Zhan Shuang Punishing attracted more than 2 million appointments from all platforms in mainland China before the public beta. What's more, on August 10, 2020, this game also linked with KFC, successfully "breaking through the fan circle" and entered the catering industry. It can be seen that cyberpunk has evolved into a special visual culture. On the Internet, there is a cyberpunk community with a huge user base; while offline, enthusiasts hold various themed exhibitions and COSPLAY events. Surprisingly, cyberpunk-themed restaurants have also appeared in the catering industry, and cyberpunk-style clothing is not uncommon. This means that this highly recognizable youth subculture is gradually spreading in all areas of our lives, and it affects the construction of youth selfidentity.

\section{FORMATION OF CYBERPUNK SUBCULTURE AND ITS VISUAL PERFORMANCE}

\subsection{Formation of Cyberpunk Visual Culture}

The word "cyberpunk" is a combination of Cybernetics and Punk, Cybernetics of which is a science that emerged in the 1930s and 1940s to study the control and communication between machines, living organisms, and organizations, and punk originated from the music subculture in the 1970s, which gradually developed into a spiritual culture later. The American science fiction industry set off the "New Wave Movement" in the 19601970s. Do Androids Dream of Electric Sheep?, a novel works of Philip K. Dick, became the pioneer of cyberpunk science fiction; In 1983, "Cyberpunk", a short story by Bruce Bosque, brought the term "Cyberpunk" into public view for the first time; In the following year, 
"Neuromancer", William Gibson's science fiction novel, came out, this novel was hailed as "the pioneering work of cyberpunk", which laid the foundation for cyberpunk science fiction at that time and later. In 1982, director Ridley Scott's sci-fi movie Blade Runner, which was adapted from Do Androids Dream of Electric Sheep?, became the originator of cyberpunk movies. It is a pity that this movie was so badly reviewed at the time, but it was later discovered its value by people over time. In addition to the Western world, Japan has also been influenced by cyberpunk. In 1988, the classic animation Akira directed by Otomo Katsuyo was released, which caused a strong social response; In 1995, the animated film Ghost in the Shell directed by Mamoru Oshii also became one of the important works in the history of cyberpunk movies. In the 21 st century, more cyberpunk works have emerged, making it more and more influential and gradually becoming a youth subculture. In addition to the examples mentioned above, some people have even transformed themselves into the "cyborg" described in the movie.

\subsection{Cyberpunk's Visual Performance}

Looking at cyberpunk movies, animations, and games, they all have almost the same visual elements. The following will summarize the characteristics of its visual performance from the two aspects of the space and color characteristics of cyberpunk.

\subsubsection{Space Characteristics}

In cyberpunk movies or animations, many tall buildings, huge holographic projection advertising screens, and dazzling neon lights are often seen, just like a high-tech utopian city. Cyberpunk cities are "super cities" built on the rapid development of science and technology, showing a kind of "out of control" phenomenon of urban development and sprawl under the background of high technology. Under the dazzling neon light effect, the steel jungle-like building clusters have created a homogeneous serialized cyber city. The buildings inside the city are replicated in a similar paradigm, forming a cluster of scientific and technological rational spaces, and becoming the image metaphor of the rectangular components in the circuit board [1].

Opposite the cyber city, it is a technological ruin of poverty and chaos. Although the high-end life that science and technology should bring, what is shown in the movie is a shabby space that is contrary to the opposite. This kind of conflict and contrast brings a visual shock to the audience, and at the same time stimulates the audience's deep thinking and imagination. In the urban space of cyberpunk movies, philosophical thinking about the development of science and technology has been integrated. In the neat, monotonous, crowded and bleak circuit board city, the future urban development picture under the motif of technological pessimism is depicted.

\subsubsection{Color Characteristics}

Cyberpunk's works have a highly recognizable visual system, that is, cold-toned, low-brightness pictures. Coupled with high-saturation and highcontrast neon lights, mysterious oriental elements, fusion of mechanical metal and punk style, cyberpunk shows a kind of fantasy and distortion beauty and mysterious appeal.

The most noteworthy is the iconic colors of cyberpunk: red, green, blue, and purple. In previous science fiction movies, blue was the main hue. It is a short-wavelength color that gives people a sense of rationality, calmness, and order psychologically, as well as a sense of spaciousness and coldness. In reality, many technology companies use blue as the main color, and the basic color of the Internet is also blue. Naturally, blue has become the dominant color in cyberpunk works full of technology. While the common colors of neon lights, another key element, are red and blue. The blue, which symbolizes technology and order, and the red, which symbolizes danger and revolution, are intermingled with each other, which is a metaphor for the disorder and anxiety in the utopian city. Moreover, green comes from electronic displays, which is also a common color in malfunction art. The cover of William Gibson's comic book Anchangel uses a large area of "electronic green." In addition, the famous cyberpunk movie The Matrix also uses green on the electronic display. Therefore, green is also one of the representative colors of cyberpunk.

\subsection{Overview of the Spiritual Core of Cyberpunk}

In numerous cyberpunk film and television games, the expression of dystopia and rebellious spirit has always been one of the themes. These rebellious behaviors can be interpreted from the following aspects: resistance to totalitarian control, 
resistance to abstinence, and resistance to instrumental rationality [2].

The resistance of the rebels against totalitarianism in cyberpunk's works stems from their struggling inner consciousness, their questioning of self-identity and their searching for the meaning of existence. This resistance is usually manifested through the actions of the characters, such as in Blade Runner, in which Dyke and Roy are killers who execute replicants for the ruler. The relationship between them and the ruler is the controller and the controlled. Because of Luo Yi's death, Dyke began to question the social identity and work nature of his "Blade Runner", and finally chose to escape.

In this type of work, the expression of the theme of "sexual liberation" is often seen, such as the kissing scene of Dyke and Rachel in the movie of Blade Runner, the sexual acts faintly visible in the dim street window in the movie of Blade Runner 2049, and the fusion of the mechanical body and the female body that Motoko Kusanagi often shows in the animation of Ghost in the Shell. The resistance to abstinence expresses the pursuit of freedom, freedom from control, and unrestrictedness. Sensibility and feeling the purest emotional relationship between people have become the most primitive pursuit in a high-tech society.

The term "instrumental rationality" originated from Marx Weber's decomposition of rationality, who decomposed rationality into "value rationality" and instrumental rationality. "Instrumental rationality" also means "purpose-reasonable behavior", while the "'purpose-reasonable behavior' refers to the behavior that can achieve the purpose on the condition that it can calculate and predict the consequences" [3]. The world constructed in the cyberpunk worldview is a world that advocates tools and technology, in which human actions are only driven by utilitarian motivations, while behaviors rely on rationality to achieve their desired goals, which are purely considered from the perspective of maximum effect, while ignoring human emotion and spiritual values. In the movie The Matrix, ordinary people become a tool to provide energy for machines, and the protagonist's behavior is precisely a resistance to human beings' refusal to become a tool of technology; In the animation Psycho-Measurer, Nobumoto Ginoza, the second leading actor, is no longer obsessed with proving himself by completing tasks due to his father's death.

\subsection{Negative Setting of Cyberpunk Works}

Cyberpunk works have constructed a gorgeous and gorgeous future ideal world for the audience, but they also unreservedly show the pessimism and cruelty in it, reflecting the fact that science and technology have solved the problem of productivity but cannot solve social contradictions. As a result, we can find that in cities under severe neon light pollution, there are still broken slums, ubiquitous drugs, crime, and sexual indulgence, and people living in them are lost and indifferent to themselves, souls, and emotions. In some cyberpunk works, the characters' rebellious behavior is demonstrated through destructive, offensive, bloody and violent behaviors. The negative images and behaviors of punk culture are integrated into the works objectively and without evasiveness. For example, in Blade Runner, Luo Yi calmly killed his "father" Taylor; in Ghost in the Shell, Kuse not only killed the researchers who had transformed himself, but also persecuted many innocent poor people. The characterization of the villain's behavior reflects the negative nature of this counterculture, which is also one point we should pay attention to when watching cyberpunk works.

\section{CYBERPUNK VISUAL CULTURE AND TEENAGERS' SELF- IDENTITY}

\section{1 "Mirror" of Cyberpunk Film and Television Works Builds the Self- identity of Teenagers}

Generally, cyberpunk movies and animations provide rich and exciting plots and various characters. During the viewing process, teenagers will naturally follow the plot to experience the character's experience, emotions, and behavioral motivations, and reflect on themselves. According to Jacques Lacan's mirror image theory, the process of adulthood will go through three stages from "ideal self" to "mirror self" and finally to "social self". Children will recognize themselves through external images such as the image of themselves in the mirror and the reflection of others around them [4]. Today, when visual text is prevalent, images of various media have formed a new "mirror" that affects people's self-construction. The most direct way for cyberpunk culture to affect young people is through the dissemination of movies, animations, and game images. The process of young people watching movies, animations, and game images is 
also a process of self-construction. Cyberpunk movies, as one of the forms of science fiction movies, allow the audience to think about the relationship between humans and "post-human" organisms, question the claims of anthropocentrism, and think about "how humans survive and how to choose What kind of person to become". The philosophical thinking aroused by the cyberpunk video works prompts the audience to actively think about issues such as survival, life, and technology. In the world of video, characters and concepts invisibly constitute the "mirror self" of young people, which will encourage young people to imitate in terms of language, behavior and values [7]. Usually, teenagers are naturally attracted by the characters in the images. This may be the character's behavior and appearance, or the character's personality, lifestyle, and so on. In the love and pursuit of such characters, teenagers will generate identification, and will express this identification based on the imitation of the language, behavior, and costume of the role in the image, and then generate the "ideal self". People can get the joy and satisfaction of babies peeking at themselves in the mirror when watching video works. If they see an image similar to them in the work, the viewer will resonate in their hearts. When a teenager completes a gaze in the mirror image of a film and television and recognizes the image in it, he will naturally be affected by these deep-seated value connotations, thereby revising or even changing his judgment on value [5].

\subsection{Cyberpunk's Consumption of Visual Symbols Affects Teenagers' Self- identity}

The social reality we live in today is an imagebased reality, and mankind has now entered the age of experience economy. The characteristic of consumption that occurs in this experience economy is visual consumption. Consumption dominates people's social and economic life, plays a driving role in the construction of social relations, and is a factor that cannot be ignored in the construction of an identity system [6]. Today, people are more inclined to perceptually feel about commodities, pay attention to the symbolic value of commodities, and selectively ignore some use values of commodities. Gradually, appearance image has become an important factor for consumers to judge whether a product is worthy of consumption. The choice of product is not only to pursue the function of the product, but also to pursue psychological satisfaction. Teenagers often purchase and use cyberpunk visual symbols to demonstrate their uniqueness, thereby affecting their self-identity, such as the purchase and use of cyberpunk-style clothing and related decorations, dressing up their personal space in cyber style, etc., these visual symbols have become a vehicle for young people to express themselves and present themselves, as well as a sign of their uniqueness.

\subsection{Participation of the Second Creation and the Virtual Communities of Cyberpunk Builds Self-identity}

The second creation of cyberpunk mainly includes the creation of offline themed cosplay activities, related film and television, and game fan works. In addition, there are also a large number of cyberpunk virtual communities on the Internet, such as related online forums, communities, groups, and circles. From July to October 2020, the game Zhan Shuang Punishing opened a character design competition, which attracted a large number of players to participate in the creation and voting. What is impressive is that it has formed a player base of more than 1.5 million on the bilibili video platform alone; The game "Cyberpunk 2077", which has not yet been released, has been discussed on Sina Weibo with more than 270,000 topics. Furthermore, onePlus mobile phone brand released "Cyberpunk 2077 Limited Edition Phone" on November 6, which caused a new round of fan carnival.

For teenagers who participate in the secondary creation of cyberpunk, users will get a positive user experience in the behavior of generating content. Self-acceptance and self-recognition of adolescents will produce positive and positive reinforcement, thereby enhancing their psychological joy and happiness. The formation of virtual communities also builds a social platform and frame of reference for group identity belonging and collective identity for young people in a critical period of selfidentification [7].

\subsection{Cyberpunk's Negative Impact on Teenagers' Self-identity}

As mentioned earlier, cyberpunk's films and games contain pornographic and violent elements in their content, which may have a negative impact on the self-identity of young people who are not yet mature. Some scholars have studied the impact of image violence on young people. They said that the more young people are exposed to online images 
containing violence, the higher their approval of violence [8]. Long-term exposure to such images may cause young people who lack discernment to subconsciously believe that violent behavior is legitimate and reasonable, which will have a negative impact on their three views and make them more likely to have the impulse to "confront society" and cause serious consequences.

It should be noted that within the spiritual core of cyberpunk, the themes of rebellion and individualism stand out, which is very much in line with the rebellious nature of adolescence. In the final analysis, the reason why young people are rebellious is due to the imbalanced physical and mental development and the chaotic external manifestations of self-identity. In fact, they hope to find answers to the questions of "who am I", "what's wrong with me", and "what should I do" through rebellion[9]. In many cyberpunk movies and animations, this is also the problem faced by the characters on the screen. After the characters could not find the answer to the question or were disappointed with the answer, a series of defiant behaviors occurred, such as Kuze in the live-action movie "Ghost in the Shell" and Kogami Shinya in the animation "Psycho-Measurer". When teenagers watch cyberpunk movies or cartoons, they are more likely to identify with the behavior and thoughts of these characters, which in turn affects them, resulting in feelings of rebellion against society, against collectivism, and loss of self.

\section{CONCLUSION}

Teenagers are at an immature stage of selfawareness and may lack judgment about works with a strong recognizable style and deep subject matter, such as cyberpunk. In receiving the communication of this kind of visual culture, through the "mirror reflection" of the screen roles, the consumer behavior of cyberpunk visual symbols and the interaction behavior of participating in the second creation and communication and virtual communities, selfpersonality is revealed and self-identity is constructed. However, due to cyberpunk's own negative assumptions, long-term exposure to such works may have some negative effects on young people. Therefore, on the basis of affirming the value of such works, we should treat this youth subculture dialectically and formulate reasonable strategies to deal with its negative effects.

As cyberpunk visual culture has formed a certain scale, it cannot be completely stripped away. First, on the basis of understanding the characteristics and spiritual core of cyberpunk, the review of such images from the source should be strengthened, and the negative factors contained in cyberpunk images should be appropriately deleted. But this does not mean that all cyberpunk works contain negative factors. Related institutions should also establish a work classification system as soon as possible to allow young people to be exposed to suitable works at different ages. Second, the characteristics of adolescents' self-identity should be fully understood. The producers of related content should shoulder certain social responsibilities and try to avoid misleading young people in the content of the work. Third, parents and educators should actively guide them to help them build up good thinking skills and three outlooks during their growth. In addition, on the basis of fully respecting young people, they help students find a balance between freedom and norms, find their direction in life, and find their true self, thereby promoting the healthy growth of young people.

\section{AUTHORS' CONTRIBUTIONS}

Qianchen Cheng is responsible for writing this paper, Kaili Xie and Shuchao Chen were responsible for the revision of the paper.

\section{REFERENCES}

[1] Zhu Mingjie. The Urban Space View of Cyberpunk Movies [J]. Contemporary Cinema, 2020(08): 41-45.

[2] Anqi Chen. Cyberpunk Movie [D]. East China Normal University, 2019.

[3] Wang Caiyun, Zheng Chao. Value Rationality and Instrumental Rationality and Their Methodological Significance — Based on Max Weber's Dichotomy of Rationality [J]. Journal of Jinan University (Social Science Edition), 2014, 24(02): 48-53.

[4] Zhang Yibing. From Narcissism to Abnormal Mirror Love: An Interpretation of Lacan's Theory of Mirror Image [J]. Tianjin Social Sciences, 2004(06): 12-18+74.

[5] Xiao Lu. Talking About the Influence of Animation on Young People from the Theory of Mirror Image [J]. Chinese Youth Research, 2012(03): 84-86. 
[6] Han Wei, Zhao Yuanlin. Research on the Commercialization of Aesthetic Interest in The Context of Visual Consumption [J]. Journal of Yulin University, 2019, 29(03): 7579.

[7] Liu Fang. The Influence of "UGC" Network Subculture on Adolescents' Self-Identity and Its Regulation [J]. Journal of Nantong University (Social Science Edition), 2019, 35(03): 106-112.

[8] Xiao Haitao. Investigation on the Impact of Video Violence on Young People [D]. Inner Mongolia University, 2013.

[9] Li Yihui. Rebellion and Adolescents' SelfIdentity - Based on the Perspective of Care Ethics [J]. Zhejiang Educational Science, 2019(04): 33-36 